\title{
Ambiente Virtual de Aprendizagem - A inclusão dos aprendizes deficientes visuais
}

\author{
Jefferson Ribeiro de Lima, Orivaldo de Lira Tavares \\ Departamento de Informática - Universidade Federal do Espírito Santo (UFES) \\ Caixa Postal 01.9011 - 29.075-910 - Vitória - ES - Brasil \\ jefferson.eadegmail.com, tavareseinf.ufes.br
}

\begin{abstract}
This article presents a proposal for adapt inclusive digital resources in the Virtual Learning Environment developed in order to assist in conducting the apprentice with visually impaired in performing the tasks and interactions that occur in the tool by means of synthesis and spoken instructions via microphone attached the computer. The learning environment designed in this paper has been tested and approved by the target audience in order to validate user autonomy in the implementation of activities without the need for assistance from others or external software.
\end{abstract}

Resumo. Este artigo apresenta uma proposta para adaptação de recursos digitais inclusivos no Ambiente Virtual de Aprendizagem desenvolvido com a finalidade de apoiar a condução do aprendiz deficiente visual na execução das tarefas e interações que ocorrem na ferramenta por meio de síntese de voz $e$ instruções pronunciadas via microfone acoplado ao computador. $O$ ambiente de aprendizagem projetado neste trabalho foi testado e homologado junto ao público alvo com o propósito de validar a autonomia do usuário na execução das atividades, sem a necessidade da ajuda de terceiros ou auxílio de softwares externos.

\section{Introdução}

Segundo os dados do Censo demográfico do IBGE (2010), mais de 45,6 milhões de brasileiros declararam ter alguma deficiência. O número representa $23,9 \%$ da população do país. A deficiência visual foi a que mais apareceu entre as respostas dos entrevistados e chegou a 35,7 milhões de pessoas.

Diante do fato que destaca o grande número de deficientes no país, uma perspectiva ideal seria oferecer soluções de aprendizagem igualitárias para todas as pessoas, sem distinção ou segregações. No entanto, a realidade atual está longe de proporcionar um atendimento uniforme que atenda a todos os aprendizes sem restrições, seja na educação presencial ou na modalidade à distância.

Contudo, as novas propostas metodológicas de aprendizagem apoiada pela concepção da Tecnologia da Informação e Comunicação (TICs) vêm transpondo barreiras com relação aos velhos modelos tradicionais de ensino, principalmente no que diz respeito à obrigatoriedade da presença física do aluno em sala de aula (Nóbrega, 2011). A partir desta compreensão, que é possível aprender em espaços diferenciados, métodos alternativos foram desenvolvidos para apoiar o educando na modalidade de Educação a Distância (EaD). O aumento crescente pela demanda de aprendizagem na 
$\mathrm{EaD}$ relaciona-se diretamente com o surgimento das aplicações categorizadas como Ambientes Virtuais de Aprendizagem (AVAs). Para Nunes et al. (2012), os AVAs são ferramentas que apresentam-se como uma opção tecnológica para atender as novas necessidades educacionais, oferecendo possibilidades de interação, compartilhamento e aquisição de novos saberes, sendo uma excelente alternativa para apoiar o ensino por meio da Internet.

No entanto, Coelho (2011) afirma que os sistemas disponibilizados para aprendizagem na $\mathrm{EaD}$ normalmente não oferecem recursos digitais inclusivos apropriados para prover acessibilidade. Estas afirmações podem ser evidenciadas através das pesquisas realizadas por Gabardo (2010), onde estudos comparativos entre os AVAs destacam carências com relação ao quesito de acessibilidade nas ferramentas analisadas, principalmente para apoiar os deficientes visuais. Diante destas evidências, nota-se a importância de propôr soluções concretas para facilitar o manuseio destas plataformas.

O público alvo foco deste trabalho são os deficientes visuais, classificados em dois grupos de indivíduos, cegos ou de baixa visão. O termo cego ou legalmente cego é usado para indicar as pessoas com ausência total de visão em decorrência de problemas congênitos ou acidentes que possivelmente provocaram danos irreversíveis na retina. Já o termo baixa visão, significa a redução da resposta visual de uma pessoa, que é determinada por uma escala que define a qualidade do campo visual. (Carvalho, 2001)

Diante do cenário apresentado, o artigo apresenta uma proposta para ampliar o acesso dos deficientes visuais, de modo a permitir a eles o uso de plataformas de ensino a distância. Para alcançar esse objetivo, foram pesquisadas e aplicadas tecnologias da atualidade no desenvolvimento de um protótipo para desempenhar ações através do reconhecimento de padrões vocais e síntese de voz. "Com intuito de garantir a inclusão devem ser asseguradas às pessoas as condições apropriadas de atendimento às peculiaridades individuais, para que possam usufruir das oportunidades existentes...". (Trindade e Garcia, 2009, p. 2).

O ambiente de aprendizagem inclusivo proposto no artigo é capaz de realizar ações apenas por instruções pronunciadas e captadas pelo microfone ligado ao computador. Os sinais de voz submetidos para o microfone serão recepcionados pelo agente assistente sendo convertidas em ações no contexto da plataforma, com o propósito de oferecer para o deficiente visual a praticidade na interação e condução da navegação sem a necessidade de auxílios adicionais.

Para oferecer um melhor detalhamento da proposta, o artigo foi dividido em 7 seções, contando com esta, a introdução. A seção 2 destaca os métodos aplicados na elaboração da pesquisa. Na seção 3 as pesquisas e os trabalhos correlatos. A seção 4 destaca as percepções dos usuários obtidas na entrevista. A seção 5 apresenta o projeto de sistema e as tecnologias aplicadas. A seção 6 destaca os resultados alcançados. Por fim, a seção 7, apresenta as considerações finais e os trabalhos futuros.

\section{Métodos Aplicados}

A pesquisa foi realizada em cinco etapas: Pesquisa em trabalhos correlatos, Entrevistas in loco em instituição de amparo ao deficiente visual para refinar a especificação dos requisitos, Projeto de sistema, Testes no protótipo e Análise dos resultados. 


\section{Pesquisa e trabalhos correlatos}

Na primeira etapa do trabalho foi efetivado um plano de investigação sobre o assunto de tecnologia assistiva direcionada para uso dos deficientes, com base nos portais $C A P E S^{1}, A_{C M}$ Digital Library ${ }^{2}$ e Google Acadêmico ${ }^{3}$, aplicando o método de Flick (2013), mapeando as contribuições dos temas averiguados e realizando análise quantitativa para obter o máximo de informações sobre o assunto.

Através dos resultados obtidos após aplicação das pesquisas, foram classificados, analisados e catalogados alguns artigos que abordam as plataformas de aprendizagem virtuais com interfaces dotadas de recursos de tecnologia assistiva (TA) para navegação e comunicação por deficientes visuais. Os trabalhos catalogados em geral, descrevem o uso dos sistemas computacionais AVAs para apoiar o aprendiz com deficiência visual.

Contudo, nota-se que o foco encontrado em grande parte das publicações, direciona-se para as interfaces tradicionais, onde toda atenção de acessibilidade é voltada para a execução das operações com apoio de programas de terceiros, como ampliadores ou leitores de tela. As aplicações ampliadoras de tela ou lupas maximizam a visualização dos textos e imagens de acordo com o posicionamento do mouse na tela. As aplicações leitoras de telas convertem as informações textuais em áudio utilizando um sintetizador de voz. (Santosa, 2009; Shinohara, 2009, Da Silva, Lückman e Wilbert, 2011; Coelho at. et., 2011; Machado, 2011; Dos Santos et AL. 2012; Franciscato e Lindemann, 2014, Carvalho, 2001).

Entre as interfaces AVAs investigadas e testadas destacamos as plataformas: Moodle e TelEduc e Amadeus. Os testes foram realizados com apoio de programas leitores de telas como NVDA ${ }^{4}$ e JAWS ${ }^{5}$ porque os ambientes de aprendizagem testados não oferecem leitores de tela inseridos na própria plataforma. No entanto os resultados alcançados com os leitores de telas escolhidos, apontaram falhas com relação a detecção e captação de algumas informações disponibilizadas nas interfaces das aplicações de aprendizagem avaliadas. Além disso, existe a necessidade da instalação dos softwares para leitura de tela no computador do usuário.

Contudo, novas soluções de TA então sendo oferecidas para realização de leitura de páginas web sem a necessidade de instalação prévia no computador. O serviço online WebAnywhere funciona deste modo, realizando a leitura sintetizada dos links e conteúdos no endereço do site fornecido pelo usuário. Para utilização do serviço, o computador deve possuir acesso à Internet e uma saída de áudio disponível. No entanto, a ferramenta não apresenta suporte para funcionar com síntese de voz para idiomas diferenciados e o seu manuseio é realizado com apoio de teclas de atalho (Bigham et al., 2008a).

\footnotetext{
Disponível em: http://www.periodicos.capes.gov.br/

Disponível em: http://dl.acm.org/

Disponível em: https://scholar.google.com.br/

NV Access. NonVisual Desktop Access (NVDA). http://www.nvda-project.org/

http://www.freedomscientific.com/products/fs/jaws-product-page.asp
} 
A proposta deste trabalho de pesquisa se diferencia das demais apresentadas porque oferece para o aprendiz deficiente visual uma alternativa que dispensa o uso de mouse, teclado e suporte de aplicações externas, como os ampliadores e leitores de telas para desempenhar ações no ambiente virtual de aprendizagem. Além disso, o sistema conta com um agente assistente que informa em tempo real sobre os novos acontecimentos que estão ocorrendo entre os pares, deixando o usuário sempre alerta sobre as interações realizadas na plataforma.

\section{Percepções dos usuários}

Para conhecer melhor a realidade do aprendiz com deficiência visual, foi realizado o levantamento de requisitos com apoio do órgão filantrópico Instituto Luiz Braille do Espírito Santo (ILBES), situado no município de Vitória. A entidade sem fins lucrativos presta serviços assistenciais às pessoas com deficiência visual.

As informações coletadas no instituto foram captadas por meio de perguntas inseridas no questionário, elaborado para identificar as necessidades gerais dos deficientes que usam a Internet para acessar os conteúdos à distância através da mediação ou não do AVA. Foram entrevistadas 25 pessoas no total, 13 do sexo masculino e 12 do sexo feminino, com diferentes graus de acuidade visual ${ }^{6}$, nas quais 15 pessoas definem-se como cegas e 10 com baixa visão.

Todos os entrevistados são maiores de idade e com nível escolaridade distribuídos do seguinte modo: 4 pessoas graduadas, 12 com ensino médio, 4 com ensino fundamental e $5 \mathrm{com}$ ensino fundamental incompletos. Entre o total dos entrevistados, 21 pessoas responderam de modo positivo para a pergunta do questionário que trata sobre a disponibilidade para realização de testes com a ferramenta desenvolvida.

No entanto, apenas 10 usuários foram selecionados para aplicação dos testes. Os critérios de seleção foram estabelecidos de acordo com o grau de instrução e a experiência prévia do voluntário com uso de softwares. Deste modo, o ensino médio foi estabelecido como a escolaridade mínima e o conhecimento prévio com uso de aplicativos para leitura de tela e navegação na web como requisitos básicos usados na seleção.

Após aplicação dos critérios estabelecidos nos dados obtidos na entrevista, foram selecionadas 10 pessoas para realização dos testes. Sendo 6 homens e 4 mulheres. Entre os selecionados, 7 definiram-se como deficientes visuais cegos e 3 com baixa visão. Entre os escolhidos, $70 \%$ dispunham de mais de duas horas por semana para participar de forma ativa nos testes e $30 \%$ ofertaram no máximo uma hora por semana. Segundo Abrahão (2009, p. 4), "O trabalho humano requer processamento de informações por aqueles que o realiza, dessa forma a compreensão de como ocorre a captação de informações torna-se um ponto relevante para a análise do trabalho."

A ideia era validar a eficácia da proposta e captar dados diretamente com os aprendizes deficientes visuais. Esse retorno foi essencial para sinalizar os possíveis rumos que a pesquisa poderia tomar e, ao mesmo tempo, indicar novos requisitos 
visando refinar a especificação e desenvolvimento, de modo a minimizar as falhas por falta de aprimoramento na proposta inicial.

\section{Projeto e Tecnologias}

Com a finalidade de demonstrar a viabilidade da proposta, foi desenvolvido um protótipo AVA que incorpora alguns recursos de uso comum em plataformas similares, com a expectativa de testar as adaptações inclusivas de acessibilidade para atender as necessidades dos aprendizes deficientes visuais. No entanto, cabe ressaltar que o ambiente sugerido é de uso coletivo para todas as pessoas. A seguir, a Figura 1 ilustra uma tela onde os recursos podem ser compartilhados através de ações convencionais, usando teclado e mouse ou apenas por instruções pronunciadas via microfone.

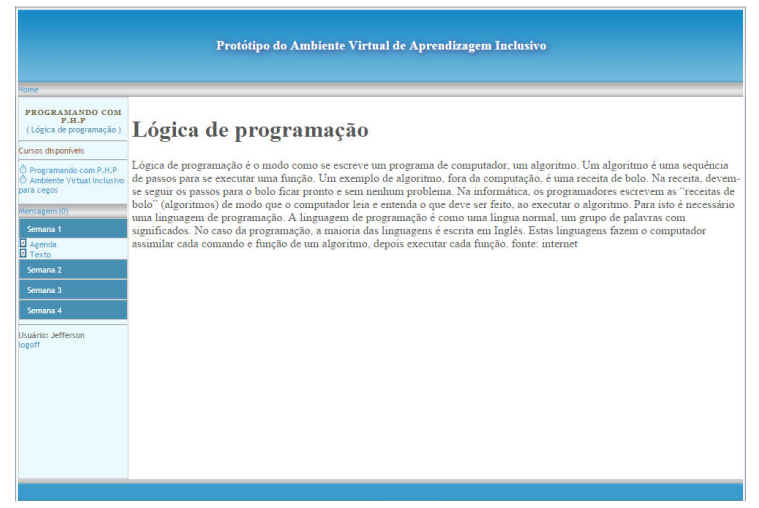

Figura 1. Tela de uso comum do protótipo AVA

Para desenvolvimento do projeto, optou-se pelo modelo incremental. Para Pressman (2006), "O modelo incremental combina elementos do modelo em cascata aplicado de maneira interativa". A diferença está em que, no modelo incremental, apenas os novos incrementos passam por todas as etapas (comunicação, planejamento, modelagem, construção e implantação), evitando dessa forma que o projeto completo percorra novamente todas as fases.

As etapas na elaboração do projeto de software foram baseadas na $U M L$. Inicialmente foram definidos os cronogramas e a especificação dos requisitos (casos de uso). Na etapa seguinte (modelagem), foram utilizadas as notações dos diagramas de classe, seqüência e estado. $\mathrm{Na}$ etapa de construção, foram definidas e aplicadas as tecnologias na produção da ferramenta. A fase de implantação foi precedida pela aplicação de testes.

\subsection{Tecnologias de software}

$\mathrm{Na}$ fase de desenvolvimento do protótipo, optou-se pelo uso da linguagem PHP (Hypertext Preprocessor) ${ }^{7}$, como plataforma base para codificação, sendo que o trabalho de persistência das informações ficou a cargo do sistema gerenciador de banco de dados $\mathrm{MySql}^{5}$. A escolha da codificação em PHP foi devida à estabilidade e simplicidade de uso semântico apresentados pela linguagem. Além disso, as ferramentas de

7 Disponível em: http://php.net/index.php. Acesso em mar. 20155 Disponível em: https://www.mysql.com. Acesso em mar. 2015 
desenvolvimento escolhidas são consolidadas no mercado, dispõem de amplo suporte na comunidade de desenvolvedores e possuem licença de uso livre.

Após estudos da escolha das tecnologias existentes que atenderiam a necessidade da interpretação dos sinais emitidos pela voz e também da sintetização da fala, optou-se pelo uso da Framework Web Speech $A P I^{8}$ da empresa Google, para operacionalizar a função de transformação das freqüências de ondas sonoras em texto, como também o inverso, texto em voz. Segundo Shire e Hans (2012), a Web Speech $A P I$ permite aos desenvolvedores na $W e b$ inserir recursos como o de reconhecimento de voz e a leitura de tela no desenvolvimento de páginas web, através de um elevado índice de abstração da complexidade que é inerente a este tipo de tecnologia de reconhecimento de padrões.

Para Yin (2005), é primordial a observação da nova tecnologia dentro de um cenário de experimentação, pois prestará uma ajuda inestimável para se compreender as vantagens e limites da tecnologia adotada. Em comparação com as alternativas similares, a tecnologia da empresa Google escolhida apresenta um nível elevado de maturidade e confiabilidade. A Web Speech API reconhece sinais de voz com diferentes timbres, sem a necessidade de realizar um prévio registro, como acorrer na maioria das ferramentas concorrentes. Além disso, dispensa instalação, pois funciona diretamente como um serviço por meio da Internet.

\subsection{Arquitetura do sistema}

A seguir, a Figura 2 apresenta a arquitetura projetada para desempenhar as ações de reconhecimento de voz e síntese de fala no AVA inclusivo. Essa arquitetura permite melhorar a acessibilidade dos aprendizes deficientes visuais em um AVA.

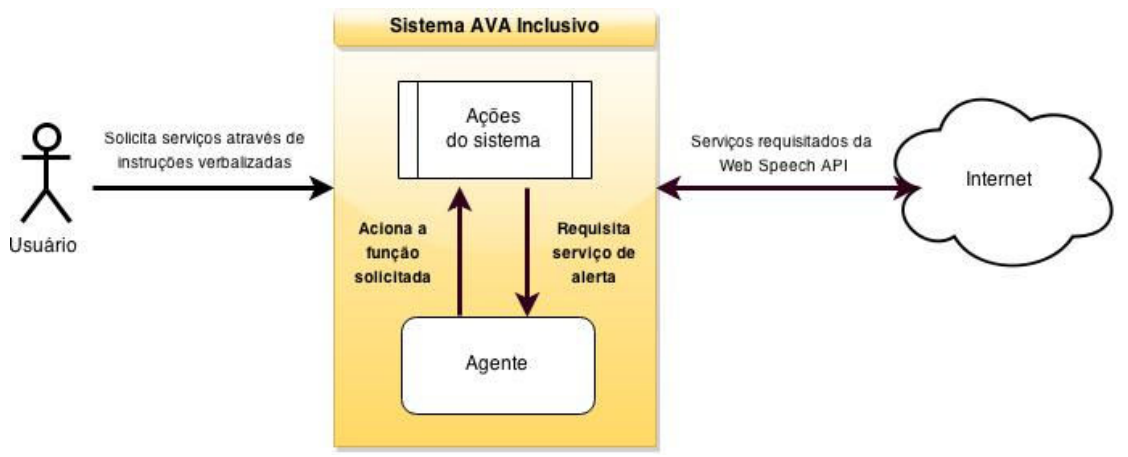

Figura 2. Esquema da arquitetura para reconhecimento de padrão vocal

A arquitetura apresentada (Figura 2) mostra o aprendiz solicitando serviços para a interface da aplicação, através de comandos verbalizados que serão captados pelo microfone, recepcionados pelo agente assistente e enviados para processamento através da Web Speech API na Internet. As solicitações processadas são retornadas em formato de texto, sendo recepcionadas pelo agente assistente do sistema que aciona as funções internas da aplicação ou anuncia, de modo sintetizado, as novas ações que estão ocorrendo em tempo real no ambiente.

Deste modo, extingue-se a dependência de uso dos softwares externos para leitura da interface da aplicação, mantendo o aprendiz sempre informado sobre sua 
condição atual no sistema. Para controlar a dinâmica das ações realizadas pelos aprendizes, o agente assistente monitora e registra as ocorrências e solicitações que ocorrem a qualquer tempo no espaço de aprendizagem, através da percepção de eventos ou entrada de dados dos usuários. Segundo Brooks (1986), um assistente possui módulos de competência, que permite respostas as requisições particulares do ambiente. O principal papel do agente assistente no AVA desenvolvido é ser os "olhos" dos aprendizes deficientes visuais, fornecendo as orientações necessárias para a condução e realização de tarefas e interações.

\subsection{Recursos}

Quando o aprendiz abre a página principal do AVA inclusivo, os dispositivos de hardware como caixas de som e microfone devem está devidamente instalados no computador. Para acionar as funcionalidades do ambiente é possível utilizar as opções convencionais da ferramenta, por meio de mouse e teclado, ou por comandos de voz predefinidos no ambiente. Desta forma, o sistema passa a conduzir a navegação apenas por instruções pronunciadas. Como padrão, o recurso que ativa a instrução por voz e o assistente está sempre com o status habilitado, conforme apresentado na Figura 3. O acesso padrão no ambiente, permite o controle e condução da aplicação apenas por comandos vocais.

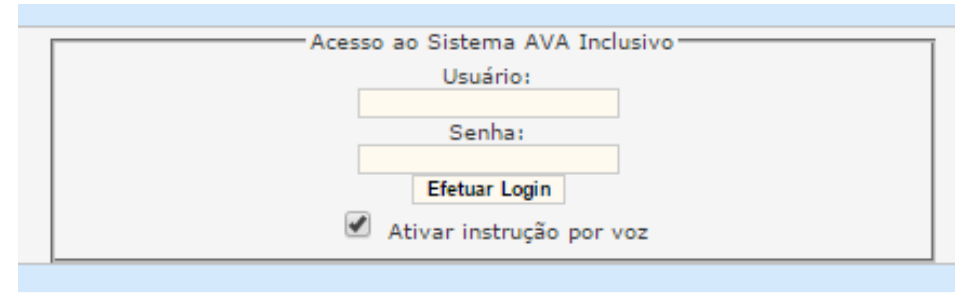

Figura 3. Tela de acesso ao AVA inclusivo

Caso o aprendiz pronuncie uma instrução inválida, o agente assistente auxilia para reiniciar o procedimento através de uma mensagem verbal de orientação. Entre os recursos digitais contemplados no desenvolvimento atual, estão incluídos: Agenda, Fóruns, Tarefas, Chat e Mensagens. O AVA apresentado neste trabalho pode ser acionado por instruções convencionais (teclado e mouse) ou vocais no sistema.

Ao acessar o AVA o sistema fica de prontidão aguardando uma nova requisição. Toda instrução pronunciada e recepcionada no AVA inclusivo, no entanto, só é realizada quando a instrução correta é fornecida. Para acionar um recurso por instrução vocal, basta pronunciar a palavra-chave Abrir seguida do comando desejado. Por exemplo, Abrir ajuda. Neste caso, o sistema orienta o usuário como desempenhar ações no ambiente da plataforma via síntese de voz. Se o comando estiver errado ou não for aceito, o sistema emite um alerta orientando para repetição dos passos. Todas as funcionalidades podem ser acionadas do mesmo modo, basta fornecer a palavra-chave. Também é possível solicitar ao sistema uma ampla gama de serviços como: orientação para navegação, listagem de usuários ativos, mensagens recebidas, tarefas realizadas e pendentes, entre outros. Além disso, a qualquer momento, é possível solicitar a lista completa dos comandos vocais predefinidos através da instrução Abrir tutorial. 


\section{Resultados Alcançados}

Com base na entrevista inicial realizada no Instituto Luiz Braille, do Espírito Santo, foi possível identificar e selecionar voluntários de acordo com os critérios definidos na seção 4 do artigo para desempenhar os testes no protótipo desenvolvido, respeitando os limites de tempo estabelecidos pelas pessoas selecionadas. Na primeira etapa, foram aplicados testes individualmente, em horários previamente agendados, com as 10 pessoas escolhidas. A idéia no primeiro momento era observar o comportamento do público alvo com relação ao uso da ferramenta, de modo a validar a proposta e, ao mesmo tempo, detectar possíveis falhas na especificação do projeto.

Para experimentação da ferramenta, o desenvolvedor/administrador do sistema criou um curso denominado: "Conhecendo o AVA inclusivo", composto por dois módulos: "Introdução ao AVA" e "Comandos básicos vocais". O primeiro módulo foi dividido em duas semanas, com atividades diversas como: leitura de texto, agenda e participação no fórum. O segundo módulo, "Comandos básicos", foi dimensionado para uma semana, incluindo uma atividade de leitura e a realização de testes pelo aprendiz. Foi necessário passar uma rápida orientação sobre os comandos básicos do sistema, para que os voluntários realizassem o primeiro acesso e utilizassem o sistema por instruções vocais.

Além disso, para verificar a qualidade da ferramenta desenvolvida, foram aplicados testes de usabilidade. A metodologia usada consiste na detecção de problemas na interação do usuário com a ferramenta, com base na apresentação antecipada dos indicadores avaliativos a serem pontuados pelos voluntários nos testes. Na execução dos experimentos, observa-se uma dificuldade inicial comumente enfrentada pelos voluntários deficientes visuais. Após abertura da página do protótipo AVA, os leitores de tela não comunicam ao usuário que o navegador está solicitando à liberação de uso do microfone, recurso essencial para uso da aplicação desenvolvida. Em todos os casos foi necessário ajudar os usuários para habilitar o microfone. Após o apoio inicial fornecido para liberação do microfone, o aprendiz conseguiu interagir com o sistema, usando apenas as instruções vocais predefinidas, e navegar entre os cursos, salas e atividades avaliativas das semanas adicionadas na simulação.

Após ingresso das pessoas no ambiente AVA inclusivo, os testes aplicados apresentaram resultados satisfatórios, com base nas afirmações dos aprendizes deficientes visuais, sendo o agente assistente fundamental para orientação, condução e realização das ações, sem intervenções externas ou auxílios adicionais. De acordo com Cybis (2007, p.7) "uma interface, segundo a perspectiva ergonômica, precisa proporcionar ao usuário a realização das tarefas de maneira eficiente, eficaz e agrádavel". Entre os 10 (dez) aprendizes selecionados para realização dos testes, 30\% dos voluntários destacaram como ponto negativo a falta de retorno imediato do agente assistente, provocando incertezas momentâneas para desempenhar ações na aplicação. Já os demais participantes (70\%), consideraram a navegação, execução e tempo de retorno satisfatório.

Após a análise do problema sobre a demora para reação do agente, identificou-se que o tempo de retorno pode variar de acordo com a velocidade de acesso à internet, ou seja, quanto mais rápida a transmissão de dados na rede, mais ágil é o retorno fornecido pelo assistente. O protótipo utiliza na execução dos processos um conjunto de serviços remotos que dependem da qualidade da transmissão, para alcançar o desempenho 
esperado. Para finalizar o teste prático, foi aplicado um novo questionário composto por cinco perguntas, com a expectativa de verificar a usabilidade do AVA desenvolvido. Foram pontuadas no questionário itens como: Acessibilidade, Autonomia, Navegação, Disponibilidade e Qualidade do produto. Com a média geral dos pontos atribuídos, o protótipo atingiu mais de $80 \%$ de aceitação no teste de usabilidade. Deste modo, conclui-se que o resultado foi positivo, sendo o protótipo aprovado pela maioria dos participantes do experimento prático. Entretanto, é importante realizar novos testes para identificar novas dificuldades e consolidar o resultado obtido. Cabe ressaltar que os experimentos foram realizados no laboratório de informática da ILBES, usando o mesmo computador portátil com microfone embutido de propriedade do desenvolvedor, conectado à Internet através da rede da instituição.

\section{Considerações finais}

$\mathrm{O}$ artigo apresentou alternativas para integrar o deficiente visual em ambientes virtuais de aprendizagem. Após a apresentação do protótipo e realização de testes, os recursos digitais de acessibilidade, adaptados ao software implementado, foram aprovados pelo público alvo.

Entre os problemas detectados, a serem resolvidos, estão a dificuldade inicial do deficiente visual em liberar o uso do microfone para utilizar as instruções vocais da aplicação e a velocidade de transmissão da Internet que influencia diretamente no tempo de resposta da aplicação.

Os próximos passos deste projeto incluem a construção de uma rotina mais segura para autenticação do usuário deficiente visual no ambiente e o acesso mobile ao AVA inclusivo, já que maioria dos entrevistados possuem telefones com acesso à Internet. Outro aperfeiçoamento futuro é incluir um agente assistente inteligente, de modo a considerar as interações prévias e o perfil do usuário no ambiente.

\section{Referências}

Abrahão, J. (1993). Ergonomia: modelos, métodos e técnicas. Trabalho apresentado no II Congresso Latino-Americano e no VI Seminário Brasileiro de Ergonomia. Florianópolis.

Bigham, J. P.; Prince, C. M.; Ladner, R. E. Addressing performance and security in a screen reading web application that enables accessibility Anywhere. In: International Conference On Web Engineering, 8., 2008, New York. - IEEE Computer Society, 2008a. p. 273-284.

Brooks, R. A. A robust layered control system for a mobile robot. In IEEE Journal of Robotics and Automation, RA-2(1986)1, 14-23.

Cybis, W., Betiol, A. H., \& Faust, R. (2007). Ergonomia e usabilidade: conhecimentos, métodos e aplicações. Novatec Editora.

Coelho, C. M. et al. Acessibilidade para pessoas com deficiência visual no Moodle. Linhas Críticas, v. 17, n. 33, p. 327-348, 2011.

Carvalho, J. O. F. Soluções tecnológicas para viabilizar o acesso do deficiente visual à Educação a Distância no Ensino Superior.Campinas: Unicamp, Dissertação de mestrado. 2001. 
Da Silva, R. A.; Lückman, A. P.; Wilbert, J. W. Acessibilidade de AVAs para o usuário PNEE: uma visão introdutória. Revista ACB: Biblioteconomia em Santa Catarina, v. 16, n. 1, p. 217-233, 2011.

Dos Santos, L. G. et al. Recursos de acessibilidade para auxiliar a navegação de estudantes cegos em um editor de diagramas. In: Anais do Simpósio Brasileiro de Informática na Educação. 2012.

Flick, U. Introdução à metodologia de pesquisa: um guia para iniciantes. Porto Alegre: Ed. Penso, 2012.

Franciscato, F. L. et al. Avaliação dos Ambientes Virtuais de Aprendizagem Moodle, TelEduc e Tidia - Ae: um estudo comparativo, PPGI/UFSM. Disponível em:< http://www.cinted.ufrgs.br/renote/dez2008/artigos/8c_fabio > Acesso em: 22 maio 2014.

Gabardo, Patricia; D. Q., Silvia R. P.; ULBRICHT, V. R. Estudo comparativo das plataformas de ensino-aprendizagem. Encontros Bibli: Revista eletrônica de biblioteconomia e ciência da informação, n. 2. sem. p. 65-84, 2010.

Instituto Brasileiro de Geografia e Estatística - IBGE. Censo 2010: Disponível em: http://www.ibge.gov.br/estadosat/temas.php?sigla=rj\&tema=censodemog2010_defic Acesso em: 16 mai. 2014.

Nóbrega, G. C. Acessibilidade aos conteúdos visuais em ambientes virtuais de aprendizagem. Revista Brasileira de Tradução Visual, v. 9, n. 9, 2011.

Nunes, C. S., Torres, M. K. L.; de Oliveira, P. C. e Nakayama, M.K. (2012) "O ambiente virtual de aprendizagem Moodle: recursos para os processos de Aprendizagem Organizacional". Em: Anais do 23 Simpósio Brasileiro de Informática na Educação, Rio de Janeiro. pp. 1-5.

Machado, D. S. Inclusão e acessibilidade: a mediação pedagógica de uma professora com deficiência visual em ambientes virtuais de aprendizagem. Revista de Educação. 2011.

Pressman, R. S. Engenharia de Software. $6^{\mathrm{a}}$ ed. Rio de Janeiro: McGraw-Hill, 2006. 40, $41,60,69,70 \mathrm{p}$.

Santarosa, L.M.C; Conforto, D.; Basso, L.O., AVA inclusivo: validação da acessibilidade na perspectiva de inter agentes com limitações visuais e auditivas. Simpósio Brasileiro de Informática na Educação, 20. Anais. Florianópolis, 2009.

Shinohara, K., \& Tenenberg, J. (2009). A blind person's interactions with technology. Communications of the ACM, 52(8), 58-66.

Shires, G., Hans, W. Web Speech API Specification. 2012. Google,Inc. Disponível em: $<$ https://dvcs.w3.org/hg/speech-api/raw-file/tip/speechapi.html\#examplesrecognition>. Acesso: 20 set. 2014.

Trindade, D. D. F. G., \& Garcia, L. S. (2013). Framework Conceitual de apoio ao Design de Ambientes Colaborativos inclusivos aos Surdos. In Anais do Simpósio Brasileiro de Informática na Educação (Vol. 24, No. 1).

Yin, Robert K. Estudos de caso: planejamento e métodos. Porto Alegre: Bookman, 2005 